\title{
Anticoagulación en pacientes con infección por SARS-CoV-2/COVID-19
}

\section{Anticoagulation in patients with SARS-CoV-2 Infection/COVID-19}

\author{
Emilio Abuabara-Franco; ; Dagoberto Serpa-Díaz'; Víctor Leal-Martínez²; Nehomar Pájaro-Galvis" José Correa-Guerrero³; \\ Jorge Rico-Fontalvo ${ }^{4}$; José Bohorquez-Rivero'; Rodrigo Daza-Arnedo²; Jonatan López-Fontalvo ${ }^{5}$; \\ Luis-Gabriel Salgado-Montiel²; María Raad-Sabaria ${ }^{1}$; Daniel Porto-Valiente ${ }^{1}$
}

Forma de citar: Abuabara-Franco E, Leal-Martínez V, Serpa-Díaz D, Pájaro-Galvis N, Correa-Guerrero J, Rico-Fontalvo $\mathrm{J}$, et al. Anticoagulación en pacientes con infección por SARS-CoV-2/COVID-19. Salud UIS. 2020; 52(4): 422-430. doi: https://doi.org/10.18273/revsal.v52n4-2020009 (c) (i)

\section{Resumen}

Introducción: el COVID-19 es una enfermedad infecciosa ocasionada por el virus RNA SARS-CoV-2, con una alta tasa de contagio y con un curso clínico heterogéneo. Existe evidencia que en fase avanzada de COVID-19 hay un riesgo aumentado de coagulación intravascular diseminada. Por lo tanto, el manejo con heparinas debe ser considerado como una estrategia terapéutica. Objetivos: nuestro objetivo principal es mostrar la literatura más relevante disponible, con referencia a la utilidad de la anticoagulación en el manejo de la hipercoagulabilidad en los pacientes con COVID-19 graves. Metodología: se realizó una revisión narrativa a partir de la búsqueda avanzada con los términos DeSC: Infecciones por Coronavirus; SARS-CoV; Coagulación Sanguínea; Coagulación Intravascular Diseminada; Disfunción orgánica múltiple; Sepsis; Síndrome de respuesta inflamatoria sistémica; Lesión renal aguda. Se utilizaron los motores de búsqueda Clinical Key, Embase, PubMed y Ovid, obteniendo un total de 143 resultados, entre artículos originales, reportes de casos, series de casos y revisiones de la literatura, se seleccionaron un total de 56 artículos que fueron utilizados para la elaboración de la presente revisión de tema. Conclusiones: el manejo con heparinas debe ser considerado como una estrategia terapéutica. Continuamos con la necesidad de futuros estudios que indaguen interrogantes en el paciente COVID-19 hospitalizado, especialmente que definan, evaluando riesgo/beneficio, que se justifica o no con respecto a la anticoagulación plena en algunos pacientes específicos con COVID-19.

Palabras clave: Infecciones por coronavirus; SARS-CoV; Coagulación sanguínea; Coagulación intravascular diseminada; Disfunción orgánica múltiple; Sepsis; Síndrome de respuesta inflamatoria sistémica; Lesión renal aguda.

\footnotetext{
1. Universidad del Sinú. Cartagena de Indias, Colombia.

2. Nuevo Hospital Bocagrande. Cartagena, Colombia.

3. Universidad de Cartagena. Cartagena de Indias, Colombia.

4. Clínica SOMA. Medellín, Colombia.

5. Clínica Antioquia. Medellín, Colombia.

Correspondencia: Nehomar Pájaro Galvis. Dirección: Transversal 54 41-117 U. Sinú, Cartagena. Teléfono: +57 3008233570. Correo electrónico: neho94@hotmail.com
} 


\begin{abstract}
Introduction: COVID-19 is an infectious disease caused by the RNA SARS-CoV-2 virus, with a high rate of infection and a heterogeneous clinical course. There is evidence that in the advanced phase of COVID-19 there is an increased risk of disseminated intravascular coagulation. Therefore, heparin management should be considered as a therapeutic strategy. Objectives: our main objective is to show the most relevant literature available, with reference to the usefulness of anticoagulation in the management of hypercoagulability in patients with COVID-19 and severe manifestations. Methodology: A narrative review was performed from the advanced search with the terms DeSC: Coronavirus Infections; SARS-CoV; Blood Coagulation; Disseminated intravascular coagulation; Multiple organic dysfunction; Sepsis; Systemic inflammatory response syndrome; Acute kidney injury. The Clinical Key, Embase, PubMed and Ovid search engines were used, obtaining a total of 143 results, among original articles, case reports, case series and literature reviews, a total of 56 articles were selected that were used to the preparation of this topic review. Conclusions: Heparin management should be considered as a therapeutic strategy. We continue with the need for future studies that investigate questions in the hospitalized COVID-19 patient, especially defining, evaluating risk / benefit, which is justified or not with respect to full anticoagulation in some specific patients with COVID-19.
\end{abstract}

Keywords: Coronavirus infections; SARS-CoV; Blood coagulation; Disseminated intravascular coagulation; Multiple organic dysfunction; Sepsis; Systemic inflammatory response syndrome; Acute kidney injury.

\section{Introducción}

Los coronavirus son una especie importante de virus RNA, que reciben su nombre por su apariencia de "corona" en la microscopia electrónica, y es capaz de producir infección en humanos y en animales. En diciembre 2019, se identificó un nuevo coronavirus que es capaz de producir neumonía e insuficiencia respiratoria, en Wuhan, provincia de Hubei en China, $\mathrm{y}$ fue nombrado SARS-CoV-2 (severe acute respiratory syndrome coronavirus 2 , por sus siglas en inglés $)^{1}$. No pasó mucho tiempo hasta generar un impacto en salud en toda China; desde luego, comenzaron a aparecer un número de casos crecientes en otros países del mundo. En febrero de 2020, la Organización Mundial de la Salud (OMS), nombró a la enfermedad que produce como "COVID-19", que significa enfermedad por coronavirus 2019². El 6 de marzo de 2020, se reportó el primer caso de COVID-19 en Colombia, una mujer 19 años proveniente de Milán, Italia ${ }^{3}$. Poco tiempo después, el 11 de marzo de 2020, la organización mundial de la salud declara al COVID-19 como una pandemia mundial ${ }^{4}$. Hasta el 3 de junio de 2020, reportaron 6416828 casos en todo el mundo, con 382867 muertes confirmadas y 216 países afectados 5 . En Colombia, a esa misma fecha, se reportaron 33354 casos, con 1045 muertes confirmados y 12288 pacientes recuperados ${ }^{6-8}$.

El SARS-CoV-2 tiene un periodo de incubación que oscila entre 2 y 14 días después de la exposición ${ }^{9}$, con una presentación clínica muy variada, desde pacientes asintomáticos, pasando por casos leves, hasta pacientes críticamente enfermos con SDRA, microtrombosis, coagulación intravascular diseminada (CID), disfunción multiorgánica y muerte ${ }^{10}$. Los factores de riesgo para el desarrollo de COVID-19 grave incluyen enfermedad pulmonar obstructiva crónica, asma moderada a severa, enfermedad cardiaca previa, obesidad, diabetes mellitus, enfermedad hepática crónica, enfermedad renal crónica en grados avanzados y pacientes inmunodeprimidos con cáncer en tratamiento con quimioterapia, antecedente de trasplante, infección por virus de inmunodeficiencia humana (VIH), uso de drogas inmunosupresoras o corticoides $^{11}$. Nuestro objetivo principal es mostrar la literatura más relevante disponible, con referencia a la utilidad de la anticoagulación en el manejo de la hipercoagulabilidad en los pacientes con COVID-19 grave.

\section{Metodología}

Se realizó una revisión narrativa a partir de la búsqueda avanzada con los términos DeSC: Infecciones por Coronavirus; SARS-CoV; Coagulación Sanguínea; Coagulación Intravascular Diseminada; Disfunción orgánica múltiple; Sepsis; Síndrome de respuesta inflamatoria sistémica; Lesión renal aguda. Se utilizaron los motores de búsqueda Clinical Key, Embase, PubMed y Ovid, obteniendo un total de 143 resultados, entre artículos originales, reportes de casos, series de casos y revisiones de la literatura, se seleccionaron un total de 56 artículos que fueron utilizados para la elaboración de la presente revisión de tema. 


\section{Hipercoagulabilidad y anticoagulación en COVID-19}

La pandemia nos ha enfrentado a un gran número de pacientes con una nueva enfermedad para la cual nadie tenía inmunidad y la ciencia aún no tiene cura. En los últimos cinco meses la enfermedad nos ha avasallado con miles de artículos que buscan desentrañar la etiología, fisiopatología y clínica para garantizar un diagnóstico y manejo oportuno, que cambien el fatal desenlace en los pacientes más críticos. El fervor desenfrenado por establecer la fisiopatología, un mecanismo causal que nos lleve a una estrategia terapéutica acertada ha llevado a la ciencia a buscar caminos conocidos y otros nunca transitados. Uno de ellos es la ya conocida vía común de la inflamación y coagulación con activación de un estado de hipercoagulabilidad ${ }^{12}$. Recientes publicaciones han mostrado una alta tasa de eventos tromboembólicos, arteriales y venosos, que podrían explicar la morbimortalidad en algunos pacientes críticos con COVID-1913,14. Lo anterior ha llevado a proponer intervenciones farmacológicas de anti-inflamatorios $\mathrm{y}$ anticoagulación. juiciosa evaluación de los riesgos contrastados con los beneficios, a la luz de la mejor evidencia.

En el paciente crítico, a pesar que su principal patrón de afección es respiratorio, se ha descrito compromiso de diferentes sistemas, dentro de los cuales están importantes trastornos hemostáticos, como es la trombocitopenia, con una prevalencia cerca del $44 \%$ de los pacientes en $\mathrm{UCI}^{15}$. Otra alteración hemostática notable es la CID, cuya presencia influye en la morbimortalidad de los pacientes críticos, por lo que se ha vuelvo un foco de atención para el desarrollo de nuevas estrategias terapéuticas ${ }^{16,17}$.

La sociedad internacional de trombosis y hemostasia (ISTH) define CID como un síndrome adquirido que se caracteriza por la activación intravascular de la coagulación con lo cual se desencadena la producción de grandes cantidades de trombina y fibrina intravascular que puede, a su vez, dañar la microvasculatura y generar disfunción multiorgánica ${ }^{18}$.

La fisiopatología de la CID clásica implica la producción sistemática de trombina intravascular, que conlleva a disfunción multiorgánica con el consecuente sangrado por consumo plaquetario, fibrinógeno y otros factores de la coagulación, además de hiperfibrinólisis secundaria. Sin embargo, existen escenarios de CID donde su condición fisiopatológica principal es la hiperfibrinolisis primaria, donde no necesariamente hay formación de coagulo, pero sí sangrado excesivo, configurando un evento catastrófico en la homeostasis, tal como se evidencia en los casos de sangrado obstétrico, leucemia promielocítica aguda y el cáncer ${ }^{19,20}$.

Hasegawa, et al. explicaron los cambios fisiopatológicos relacionados con la cascada de la coagulación que se describían en pacientes con sindróme de distress respiratorio del adulto (SDRA). En su investigación demostraron que el $26 \%$ de los pacientes con SDRA, incluyendo a los pacientes con sepsis que desarrollan SDRA (con una prevalencia del $40 \%$ ), presenta coagulopatía que impactan en los días de estancia en UCI y tiempo de ventilación mecánica ${ }^{21}$.

Las complicaciones hematológicas en los pacientes con infecciones de tipo viral se han descrito a través del tiempo. En 1999 fue publicado un artículo de revisión donde se describieron las características clínicas y hematológicas que presentaban este tipo de pacientes, siendo esto un punto de inflexión positivo en la curva de conocimiento que se viene adelantando vertiginosamente en este campo ${ }^{22}$. Para esto es necesario traer a colación la revisión publicada por Yang y Tang. donde se plantea la hipótesis sobre el impacto de los procesos infecciosos, dado entre otros por la aberrante activación de células endoteliales, sobre la homeostásis de la coagulación ${ }^{23}$. Con base en ello, debería tenerse en cuenta que los pacientes con infección por SARSCoV-2 pueden cursar con tiempos de protrombina prolongado y/o dímero D elevado, asociandose con mal pronóstico, desarrollo de SDRA y alta mortalidad ${ }^{24-26}$.

El incremento del dimero D, se presenta por la proteólisis de la fibrina extravasada del epitelio alveolocapilar secundario al proceso inflamatorio inducido por la infección del SARS-CoV-2. En el instante en el que el virus infecta el epitelio alveolar se genera activación de macrófagos alveolares, quimiotaxis de neutrófilos mediado por citoquinas y activación plaquetaria con la subsecuente formación de fibrina. En el momento de la lesion endotelial, los neutrófilos activados fagocitan al virus, pero también atrapan la fibrina extravasada, generando así proteólisis de la misma y como resultado elevación sérica del dímero $\mathrm{D}^{27-29}$.

En consecuencia, a lo anterior descrito, se podría disernir que el mecanísmo fisiopatológico por el cual se afecta el sistema respiratorio y hematológico, es secundario la activación de los altos niveles de citoquinas proinflamatorias (IL-2, IL-7, factor estimulador de 
granulocitos, MCP1, MIP1A, IP10 y FNT-a), las cuales inducen la triada de Virchow: estado hipercoagulable, injuria endotelial y estasis del flujo sanguíneo. Estos hallazgos se han encontrando ampliamente en los pacientes hospitalizados en unidad de cuidados intensivos ${ }^{30}$. El sistema respiratorio también exhibe consecuencias de la traida de Virchow, como es el daño alveolar difuso que conlleva al SDRA producido por el SARS-COV, el cual se podría explicar por la fibrinolisis secundaria, asociado a un incremento en la actividad plasminógeno-plasmina y el posterior aumento en los depósitos alveolares de fibrina. Esta evidencia se obtuvo de estudios preliminares en modelos animales infectados por SARS-CoV y actualmente se sugiere que podrían compartir el mismo mecanísmo fisiopatológico con el actual SARS-CoV-2 ${ }^{31}$.

En una serie de casos reportados por Yao, et al. histológicamente demostraron la presencia de microtrombos asociados a lesión alveolar difusa en pacientes con SDRA por COVID-19, sumado a la presencia de microtrombos glomerulares a nivel renal ${ }^{32}$. Entonces aparece el concepto de tromboinflamación, el cual se refiere a la íntima interacción que existe entre las diferentes vías o mecanismos de inflamación que incluyen a la IL6, complemento, PAMPs, NETs, polifosfatos y otras citoquinas, con la subsecuente activación de los factores de la coagulación y la eventual micro o macrotrombosis ${ }^{33,34}$.

Curiosamente y diferente a otras entidades infecciosas, la coagulopatía asociada a COVID-19 consumé poco los factores de la coagulación, con niveles normales o elevados de fibrinógeno y ocasionalmente trombocitopenia, por lo que sugiere un perfil trombogénico contrario a la CID, donde predomina más la fibrinólisis y una tendencia a la hemorragia, y que se presenta en aproximadamente el $38 \%$ de los pacientes críticos con COVID-19 $9^{35,36}$.

Este perfil trombogénico ha llevado al planteamiento de multiples mecanismos fisiopatológicos que expliquen esta entidad. Obviamente la inflamación endotelial es uno de ellos, generando altos niveles de antígeno del factor de von Willebrand (vWF:Ag) y el factor de coagulación VIII (FVIII), generando una cascada procoagulante. La vasoconstricción secundaria a la hipoxemia en los capilares pulmonares, conlleva a la reducción del flujo sanguíneo y oclusión vascular ${ }^{37}$. En recientes publicaciones sugieren la activación del complemento como un mecanismo procoagulante asociado, y se presenta en respuesta a la lesión endotelial, generando niveles altos de C5a y así promoviendo la activación del factor tisular y su unión al factor VII (F. VII), aumentando los niveles del factor $\mathrm{Xa}$, trombina y fibrina, configurando la triada de Virchow antes descrita $^{38}$. Prueba de ello, en un reporte de casos se encontró lesiones tromboembólicas microvasculares generalizadas que afectaban los pulmones y la piel, y estas estaban mediadas por el complemento. En dichas lesiones se aisló grandes depósitos del complejo $\mathrm{C} 5 \mathrm{~b}$ 9, C4b y MASP2, siendo el primero una característica que comparte con síndromes microtromboticos como el síndrome hemolítico urémico, síndrome antifosfolípidos y la purpura fulminante ${ }^{39-42}$.

Por todo lo anteriormente descrito, la infección viral por SARS-CoV-2 en la fase grave de la enfermedad, se asocia con coagulopatía, exhibiendo un comportamiento clínico y paraclínicos similar a la coagulopatía inducida por sepsis. Si bien, la incidencia de enfermedad tromboembólica venosa (TEV) en la neumonía grave por SARS-COV2 está alrededor del $25 \%$, y que no difiere de la incidencia de TEV en los pacientes críticos (alrededor del 13 al $30 \%$ ), cabe resaltar que un registro reciente reportó una tasa de incidencia del casi el $70 \%$ de trombosis venosa profunda periférica (TVP) y del $23 \%$ de embolia pulmonar $(\mathrm{EP})^{43-44}$.

Una cohorte prospectiva en pacientes con COVID-19 que ingresaban a UCI por falla respiratoria aguda hipoxémica, evidenció una alta prevalencia de tromboembolismo pulmonar $(16,7 \%)$ a los pocos días de su estancia, a pesar de tener tratamiento anticoagulante profiláctico o anticoagulación a dosis plena $^{45}$. Durante un estudio en China, donde se realizó el score de Padua para evaluar el riesgo tromboembólico, se encontró que los pacientes con COVID-19 con alto riesgo tenían un pobre pronóstico comparado con los pacientes con bajo riesgo ${ }^{46}$. Todo lo anterior sugiere la necesidad de estrategias diagnósticas dirigidas a la detección temprana y así al tratamiento oportuno de complicaciones tromboembólicas en los pacientes con SARS-CoV-2 ${ }^{47}$.

Otras complicaciones tromboembólicas que cabe mencionar en los pacientes con COVID-19, son el accidente cerebrovascular isquémico y síndrome coronario agudo, aunque estos son de menor frecuencia comparados con la embolia pulmonar y TEV periférico ${ }^{35,48}$. En un reciente reporte de caso, se describió el priapismo como otra posible complicación tromboembólica en pacientes con COVID-19, y que requirió manejo emergente (Aspiración de sangre cavernosa e inyección de un agente simpaticomimético a nivel intracavernoso $)^{49}$. 
Por estas razones, la tromboprofilaxis rutinaria y la anticoagulación, son estrategias a considerar en el arsenal terapéutico de esta enfermedad, equilibrando el riesgo de sangrado. Se sabe que la tromboprofilaxis en pacientes críticamente enfermos reduce hasta el $50 \%$ los eventos tromboembólicos venosos ${ }^{50,51}$.

Los resultados iniciales indican un posible beneficio clínico con las heparinas de bajo peso molecular (HBPM) en las infecciones graves por COVID-19. Tang, et al. en un estudio retrospectivo de 449 pacientes, evaluaron la intervención con HBPM o heparina no fraccionada (HNF) y su impacto en la supervivencia a los 28 días. Así, el uso de ésta estrategia terapéutica tiene un efecto protector en la supervivencia (OR: 0.44 IC $(0,22-0,85), p=0,017)$, logrando su mayor impacto en pacientes con dimero D mayor de 6 veces el límite superior de la normalidad (LSN) y un score $\mathrm{SIC}>_{\mathrm{O}}=4^{52}$. La escala $\mathrm{SIC}$, de sus siglas en inglés (Sepsis-induced coagulopathy), fue validada por Iba, et al. con el objetivo de predecir una CID inducida por ${ }^{28}$. Por ende, se resalta la importancia de una intervención terapéutica temprana con anticoagulantes, donde ha demostrado mayor beneficio (ver Figura 1 adaptada de recomendaciones en china y EEUU $)^{53,54}$.

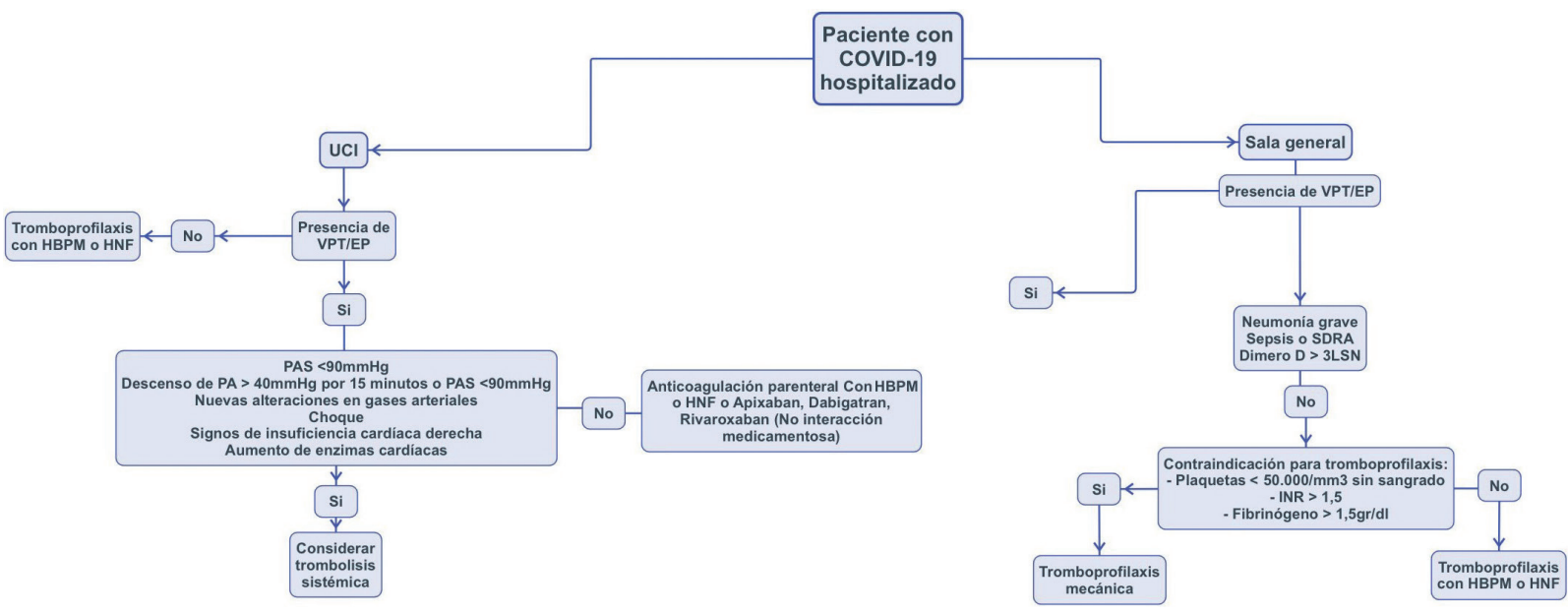

Figura 1. Algoritmo intervención terapéutica.

En 4 registros clínicos con un total 387 pacientes con COVID-19, donde se tenía como objetivo relacionar el dímero $\mathrm{D}$, conteo de plaquetas y tiempo de protrombina en presencia de una condición severa de la enfermedad, se evidenció claramente la asociación de niveles elevados de dímero $\mathrm{D}$ con la severidad de dicho cuadro clínico. No obstante, no fue posible relacionar las variables anteriores con los niveles de plaquetas y tiempo de protrombina, puesto que estos pacientes manejaron valores normales independientemente de la gravedad de la infección (ver Tabla 1) ${ }^{25,55-57)}$. Sumado a la evidencia descrita, una revisión sistemática de 9 estudios clínicos realizada por Violi, et al. encontraron una relación entre los niveles de dímero $\mathrm{D}$ elevados y la mortalidad de los pacientes con COVID-19, no siendo así con el recuento de plaquetas, tiempo de protrombina y la gravedad del espectro clínico ${ }^{58}$.

Tabla 1. Dímero D, conteo de plaquetas y tiempo protrombina en pacientes con enfermedad severa.

\begin{tabular}{lccc}
\hline \multicolumn{1}{c}{ Estudios } & Dímero D & Plaquetas & Tiempo protrombina \\
\hline Huang, et al (2020) n:41 (20) & $2,4(0,6-14,4)$ & $196(165-263)$ & $12,2(11,2-13,4)$ \\
Zhou, et al (2020) n:191 (3) & $5,2(1,5-21,1)$ & $165,5(107,0-229,0)$ & $12,1(11,2-13,7)$ \\
Wu, et al (2020) n:117 (2) & $1,16(0,46-5,37)$ & $187,00(124,50-252,50)$ & $11,70(11,10-12,45)$ \\
Wang, et al (2020) n:138 (21) & $0,4(0,2-13,2)$ & $142(110-202$ & $13,2(12,3-14,5)$ \\
\hline
\end{tabular}




\section{Tratamiento}

A pesar de la poca evidencia existente con alto nivel estadístico del beneficio absoluto de la terápia anticoagulante y tromboprofilaxis, contamos con investigaciones que apoyan esta hipótesis, como lo demostró Tang, et al. ${ }^{52}$ Adicionalmente las guías de manejo recomendan la anticoagulación o tromboprofilaxis sobre la ausencia de tratamiento ${ }^{59-61}$.

En consecuencia, en todos pacientes hospitalizados en sala general y unidad de cuidados intensivos, en ausencia de complicaciones tromboembolicas y contraindicaciones para la terapia, se recomienda la tromboprofilaxis con heparina de bajo peso molecular, heparina no fraccionada y el fondaparinux ${ }^{61}$. Cabe resaltar, que existe preferencia sobre la heparina de bajo peso molecular y la heparina no fraccionada frente el fondaparinux, sobre todo en el paciente crítico $^{59,60}$. No se recomienda el manejo antiplaquetario como prevención de $\mathrm{TEV}^{61}$.

En pacientes sin evidencia clínica de TVP o PE, no se recomienda la tromboprofilaxis ambulatoria al momento del alta. Sin embargo, se debe considerar tromboprofilaxis en pacientes con bajo riesgo de sangrado y alto riesgo de TEV, dado por Padua score $>\mathrm{o}=4$, dimedo $\mathrm{D}>3000 \mathrm{ng} / \mathrm{ml}$ o que haya cursado con formas graves de COVID-19 asociado a alguna de las siguientes: ferritina $>1000 \mathrm{ng} / \mathrm{ml}$, IL-6 $>40 \mathrm{pg} / \mathrm{ml}$, linfopenia $<800 / \mathrm{mm} 3$ y dimero D $>1500 \mathrm{ng} / \mathrm{ml}$. En los pacientes con TVP/EP confirmado, sin interección medicamentosa, se sugiere la anticoagulación con apixaban, dabigatran o rivaroxaban por 3 meses $^{61}$.

En los casos de diagnóstico positivo para TVP/ EP en pacientes en hospitalización general o UCI, sin choque o falla cardíaca derecha, se sugiere la anticoagulación parenteral con heparina de bajo peso molecular o heparina no fraccionada. Los pacientes con complicaciones tromboembólicas asociadas a choque o falla cardiaca derecha, se recomienda la trombolisis sistémica. En la administración de la terapia trombolítica se prefiere el uso de un acceso periférico frente al acceso central ${ }^{61}$.

Se aconseja no realizar la búsqueda activa de complicaciones tromboembólicas en pacientes asintomáticos o sin sospechas de EP. En los pacientes con episodios de TVP/EP recurrente con COVID-19, a pesar del tratamiento anticoagulante con heparina de bajo peso molecular, se recomienda aumentar la dosis en un 25 al $30 \%$. En los casos de anticoagulación con anticoagulantes orales y persistencia de complicaciones tromboembólicas, se sugiere el cambio a esquema con heparina de bajo peso molecular ${ }^{61}$.

En la Figura 1 abajo planteamos un algoritmo de abordaje de pacientes con COVID-19 dependiendo de su estancia hospitalaria y la presencia de TVP y/o EP. En las Tablas 2 y $\mathbf{3}$ se describen las dosis profilácticas de heparina de bajo peso molecular.

Tabla 2. Dosis profiláctica heparina de bajo peso molecular según función renal.

\begin{tabular}{lcc}
\hline & & Función renal \\
\cline { 2 - 3 } Dosis & Aclaramiento de creatinina $>30 \mathrm{~mL} / \mathrm{min}$ & Aclaramiento de creatinina $<30 \mathrm{~mL} / \mathrm{min}$ \\
Profilácticas & $<80 \mathrm{Kg}: 40 \mathrm{mg} / 24 \mathrm{~h} \mathrm{s.c}$ & $<80 \mathrm{Kg}: 20 \mathrm{mg} / 24 \mathrm{~h} \mathrm{s.c}$ \\
Enoxaprina & $80-100 \mathrm{Kg}: 60 \mathrm{mg} / 24 \mathrm{~h} \mathrm{s.c}$ & $>80 \mathrm{Kg}: 40 \mathrm{mg} / 24 \mathrm{~h}$ s.c \\
& $>100 \mathrm{Kg}: 40 \mathrm{mg} / 12 \mathrm{~h} \mathrm{s.c}$ & \\
\hline
\end{tabular}

Tabla 3. Dosis profiláctica heparina de bajo peso molecular en pacientes con riesgo de sangrado mayor.

\begin{tabular}{lll}
\hline Dosis & \multicolumn{2}{c}{ Función renal } \\
\cline { 2 - 3 } $\begin{array}{l}\text { Profilácticas } \\
\text { para pacientes } \\
\begin{array}{l}\text { con riesgo de sangrado mayor } \\
\text { Enoxaprina }\end{array}\end{array}$ & Aclaramiento de creatinina $>30 \mathrm{~mL} / \mathrm{min}$ & Aclaramiento de creatinina $<30 \mathrm{~mL} / \mathrm{min}$ \\
\hline
\end{tabular}

\section{Conclusión}

En conclusión, existe evidencia que en fase avanzada de COVID-19 hay un riesgo aumentado de coagulación intravascular diseminada, parecido a lo observado en la coagulopatía inducida por sepsis, pero con un fenotipo predominantemente trombótico. Por lo tanto, el manejo con heparinas debe ser considerado como una estrategia terapéutica. Continuamos con la necesidad de futuros estudios que indagen interrogantes en el paciente COVID-19 hospitalizado, especialmente que definan, evaluando riesgo/beneficio, que se justifica o no con respecto a la anticoagulación plena en algunos pacientes específicos con COVID-19. 


\section{Referencias}

1. Coronaviridae Study Group of the International Committee on Taxonomy of Viruses. The species Severe acute respiratory syndrome-related coronavirus: classifying $2019-\mathrm{nCoV}$ and naming it SARS-CoV-2. Nat Microbiol. 2020; 5(4): 536-544. doi: https://doi.org/10.1038/s41564-020-0695-z

2. Zhong J, Tang J, Ye C, Dong L. The immunology of COVID-19: is immune modulation an option for treatment? Lancet Rheumat. 2020; S266599132030120X.

3. Ministerio de Salud y Protección Social. Colombia confirma su primer caso de COVID-19. 6 de marzo de 2020; https://www.minsalud.gov.co/Paginas/ Colombia-confirma-su-primer-caso-de-COVID-19. aspx.

4. World Health Organization. Coronavirus disease 2019 (COVID-19) Situation Report - 51. 11 de marzo de 2020.

5. World Health Organization. Coronavirus disease (COVID-19) outbreak situation. 3 de junio de 2020.

6. González C, Yama E, Yomayusa M, VargasJ, Rico J, Ariza A, et al. Consenso colombiano de expertos sobre recomendaciones informadas en la evidencia para la prevención, diagnóstico y manejo de la lesión renal aguda por SARS-CoV-2/COVID-19. Rev Colomb Nefrol. 2020; 7(Supl 2). doi: https:// doi.org/10.22265/acnef.7.Supl.2.473

7. Daza Arnedo R, Aroca Martínez G, Rico Fontalvo JE, Rey Vela E, Pájaro Galvis NE, Salgado Montiel LG, et al. Terapias de purificación sanguínea en COVID-19. Rev Colomb Nefrol. 2020; 7(Supl.2). doi: https://doi.org/10.22265/acnef.7.Supl.2.476

8. Ministerio de Salud y Protección social, Instituto nacional de salud. Situación actual de Nuevo Coronarivus (COVID-19). 3 de junio de 2020; https://d2jsqrio60m94k.cloudfront.net/.

9. Richardson S, Hirsch JS, Narasimhan M, Crawford JM, McGinn T, Davidson KW, et al. Presenting characteristics, comorbidities, and outcomes among 5700 patients hospitalized with COVID-19 in the New York City Area. JAMA. 2020; 323(20): 20522059. doi: 10.1001/jama.2020.6775

10. Shang J, Ye G, Shi K, Wan Y, Luo C, Aihara H, et al. Structural basis of receptor recognition by SARSCoV-2. 2020; 581(7807): 221-224. doi: 10.1038/ s41586-020-2179-y

11. Moore JB, June CH. Cytokine release syndrome in severe COVID-19. Science. 2020; 368(6490): 473474. doi: 10.1126/science.abb8925
12. Leisman DE, Deutschman CS, Legrand M. Facing COVID-19 in the ICU: vascular dysfunction, thrombosis, and dysregulated inflammation. Intensive Care Med. 2020;46(6): 1105-1108. doi: 10.1007/s00134-020-06059-6

13. Clerkin KJ, Fried JA, Raikhelkar J, Sayer G, Griffin JM, Masoumi A, et al. COVID-19 and Cardiovascular Disease. Circulation. 2020; 141(20): 1648-1655. doi: https://doi.org/10.1161/ CIRCULATIONAHA.120.046941

14. Ciceri F, Beretta L, Scandroglio AM, Colombo S, Landoni $G$, Ruggeri A, et al. Microvascular COVID-19 lung vessels obstructive thromboinflammatory syndrome (MicroCLOTS): an atypical acute respiratory distress syndrome working hypothesis. Crit Care Resusc. 2020; 22(2): 95-97.

15. Williamson DR, Albert M, Heels-Ansdell D, Arnold DM, Lauzier F, Zarychanski R, et al. Thrombocytopenia in critically ill patients receiving thromboprophylaxis: Frequency, risk factors, and outcomes. Chest. 2013; 144(4): 1207-1215. doi: 10.1378/chest.13-0121

16. Selvin E, Lazo M, Chen Y, Shen L, Rubin J, McEvoy JW, et al. Diabetes mellitus, prediabetes, and incidence of subclinical myocardial damage. Circulation. 2014; 130(16): 1374-1382. doi: 10.1161/CIRCULATIONAHA.114.010815

17. Wang D, Hu B, Hu C, Zhu F, Liu X, Zhang J, et al. Clinical characteristics of 138 hospitalized patients with 2019 novel coronavirus-infected pneumonia in Wuhan, China. JAMA. 2020; 323(11): 1061-1069. doi: 10.1001/jama.2020.1585

18. Iba T, Di Nisio M, Levy JH, Kitamura N, Thachil J. New criteria for sepsis-induced coagulopathy (SIC) following the revised sepsis definition: A retrospective analysis of a nationwide survey. BMJ Open. 2017; 7(9): 1-8. doi: 10.1136/ bmjopen-2017-017046

19. Sarup BM. Diagnosis and treatment of disseminated intravascular coagulation (DIC). J Assoc Physicians India. 1973; 21(10): 861-866.

20. Levi M, Scully M. How I treat disseminated intravascular coagulation. Blood. 2018; 131(8): 845-854. doi: 10.1182/blood-2017-10-804096

21. Hasegawa N, Husari AW, Hart WT, Kandra TG, Raffin TA. Role of the coagulation system in ARDS. Chest. 1994; 105(1): 268-277. doi: 10.1378/ chest.105.1.268 
22. Van Gorp ECM, Suharti C, ten Cate H, Dolmans WMV, van der Meer JWM, ten Cate JW, et al. Review: Infectious diseases and coagulation disorders. J Infect Dis. 1999; 180(1): 176-186. doi: 10.1086/314829

23. Yang $Y$, Tang H. Aberrant coagulation causes a hyper-inflammatory response in severe influenza pneumonia. Cell Mol Immunol. 2016; 13(4): 432442. doi: 10.1038/cmi.2016.1

24. Cao W, Li T. COVID-19: towards understanding of pathogenesis. Cell Res. 2020; 30(5): 367-369. doi: 10.1038/s41422-020-0327-4

25. Wu C, Chen X, Cai Y, Xia J, Zhou X, Xu S, et al. Risk Factors associated with acute respiratory distress syndrome and death in patients with coronavirus disease 2019 Pneumonia in Wuhan, China. JAMA Intern Med. 2020; 180(7): 1-11. doi: 10.1001/jamainternmed.2020.0994

26. Tang N, Li D, Wang X, Sun Z. Abnormal coagulation parameters are associated with poor prognosis in patients with novel coronavirus pneumonia. J Thromb Haemost. 2020; 18(4): 844-847. doi: 10.1111/jth.14768

27. Xu Z, Shi L, Wang Y, Zhang J, Huang L, Zhang C, et al. Pathological findings of COVID-19 associated with acute respiratory distress syndrome. Lancet Respir Med. abril de 2020; 8(4):420-422. doi: 10.1016/S2213-2600(20)30076-X

28. Iba T, Levi M, Levy JH. Sepsis-Induced coagulopathy and disseminated intravascular coagulation. Semin Thromb Hemost. 2020; 46(1): 89-95. doi: 10.1055/s-0039-1694995

29. Najem MY, Couturaud F, Lemarié CA. Cytokine and chemokine regulation of venous thromboembolism. J Thromb Haemost. 2020; 18(5): 1009-1019. doi: https://doi.org/10.1111/jth.14759

30. Lillicrap D. Disseminated intravascular coagulation in patients with 2019-nCoV pneumonia. J Thromb Haemost. 2020; 18(4): 786-787. doi: 10.1111/ jth. 14781

31. Gralinski LE, Iii AB, Jeng S. Mechanisms of severe acute respiratory syndrome coronavirus-. MBio. 2013; 4(4): 1-12. doi: 10.1128/mBio.00271-13

32. Yao XH, Li TY, He ZC, Ping YF, Liu HW, Yu SC, et al. A pathological report of three COVID-19 cases by minimally invasive autopsies. Zhonghua Bing Li Xue Za Zhi. 2020; 49(5): 411-417. doi: 10.3760/ cma.j.cn112151-20200312-00193

33. Connors J, Levy JH. COVID-19 and its implications for thrombosis and anticoagulation COVID-19 and its implications for thrombosis and anticoagulation. Blood. 2020; 135(23): 2033-2040. doi: 10.1182/ blood.2020006000
34. Connors JM, Levy JH. Thromboinflammation and the hypercoagulability of COVID-19. J Thromb Haemost. 2020; 18(7): 1559-1561. doi: 10.1111/ jth.14849

35. Klok FA, Kruip MJHA, van der Meer NJM, Arbous MS, Gommers DAMPJ, Kant KM, et al. Incidence of thrombotic complications in critically ill ICU patients with COVID-19. Thromb Res. 2020; 191: 145-147. doi: https://doi.org/10.1016/j. thromres.2020.04.013

36. Fan BE, Chong VCL, Chan SSW, Lim GH, Lim $\mathrm{KGE}$, Tan GB, et al. Hematologic parameters in patients with COVID-19 infection. Am J Hematol. 2020; 95(6). E131-E134. doi: 10.1002/ajh.25774

37. Yan SF, Mackman N, Kisiel W, Stern DM, Pinsky DJ. Hypoxia/hypoxemia-induced activation of the procoagulant pathways and the pathogenesis of ischemia-associated thrombosis. Arterioscler Thromb Vasc Biol. 1999; 19(9): 2029-2035. doi: 10.1161/01.atv.19.9.2029

38. Chauhan AJ, Wiffen LJ, Brown TP. COVID-19: a collision of complement, coagulation and inflammatory pathways. $\mathrm{J}$ Thromb Haemost. 2020; published ahead of print. doi: https://doi. org/10.1111/jth.14981

39. Magro CM, Momtahen S, Mulvey JJ, PhD, Yassin AH, Kaplan RB, Laurence JC. Role of the skin biopsy in the diagnosis of atypical hemolytic uremic syndrome. Am J Dermatopathol 2015; 37(5): 349359. doi: 10.1097/DAD.0000000000000234

40. Campbell CM, Kahwash R. Will complement inhibition be the new target in treating COVID-19related systemic thrombosis? Circulation. 2020; 141(22): 1739-1741. doi: 10.1161/ CIRCULATIONAHA.120.047419

41. Magro C, Mulvey JJ, Berlin D, Nuovo G, Salvatore S, Harp J, et al. Complement associated microvascular injury and thrombosis in the pathogenesis of severe COVID-19 infection: A report of five cases. Transl Res. 2020; 220: 1-13. doi: 10.1016/j.trs1.2020.04.007

42. Magro CM, Harmanc AP, Klingerb D, Oroszd C, Adamsd P, Waldman J, et al. Use of C4d as a diagnostic adjunct in lung allograft biopsies. Am J Transplant. 2003; 3(9): 1143-1154. doi: 10.1034/j.1600-6143.2003.00152.x

43. Cui S, Chen S, Li X, Liu S, Wang F. Prevalence of venous thromboembolism in patients with severe novel coronavirus pneumonia. J Thromb Haemost. 2020. doi: 10.1111/jth.14830 [Epub ahead of print] 
44. Llitjos JF, Leclerc M, Chochois C, Monsallier JM, Ramakers M, Auvray M, et al. High incidence of venous thromboembolic events in anticoagulated severe COVID-19 patients. J Thromb Haemost. 2020; 18(7): 1743-1746. doi: 10.1111/jth.14869

45. Helms J, Tacquard C, Severac F, Leonard-Lorant I, Ohana M, Delabranche X, et al. High risk of thrombosis in patients with severe SARS-CoV-2 infection: a multicenter prospective cohort study. Intensive Care Med. 2020; 46(6): 1089-1098. doi: 10.1007/s00134-020-06062-x

46. Wang T, Chen R, Liu C, Liang W, Guan W, Tang R, et al. Attention should be paid to venous thromboembolism prophylaxis in the management of COVID-19. Lancet Haematol. 2020; 7(5): e362-e363. doi: 10.1016/S2352-3026(20)30109-5

47. Lodigiani C, Iapichino G, Carenzo L, Cecconi M, Ferrazzi P, Sebastian T, et al. Venous and arterial thromboembolic complications in COVID-19 patients admitted to an academic hospital in Milan, Italy. Thromb Res. 2020; 191: 9-14. doi: 10.1016/j. thromres.2020.04.024

48. Bikdeli B, Madhavan MV, Jimenez D, Chuich T, Dreyfus I, Driggin E, et al. COVID-19 and thrombotic or thromboembolic disease: implications for prevention, antithrombotic therapy, and followup. J Am Coll Cardiol. 2020; 75(23): 2950-2973. doi: 10.1016/j.jacc.2020.04.031

49. Lamamri M, Chebbi A, Mamane J, Abbad S, Munuzzolini M, Sarfati F, et al. Priapism in a patient with coronavirus disease 2019 (COVID-19): A case report. Am J Emerg Med. 2020; S0735675720305143. https://linkinghub.elsevier. $\mathrm{com} /$ retrieve/pii/S0735675720305143

50. Rosovsky R, Zhao K, Sista A, Rivera-Lebron B, Kabrhel C. Pulmonary embolism response teams: Purpose, evidence for efficacy, and future research directions. Res Pract Thromb Haemost. 2019; 3(3): 315-330. doi: 10.1002/rth2.12216

51. Alhazzani W, Lim W, Jaeschke RZ, Murad MH, Cade J, Cook DJ. Heparin thromboprophylaxis in medical-surgical critically ill patients: a systematic review and meta-analysis of randomized trials. Crit Care Med. 2013; 41(9): 2088-2098. doi: 10.1097/ CCM.0b013e31828cf104.

52. Tang N, Bai H, Chen X, Gong J, Li D, Sun Z. Anticoagulant treatment is associated with decreased mortality in severe coronavirus disease 2019 patients with coagulopathy. J Thromb Haemost. 2020; 18(5): 1094-1099. doi: $10.1111 /$ jth. 14817
53. Huang X, Chinese Society of Neurology, Peripheral Neuropathy Collaboration Group of Chinese Society of Neurology, Chinese Society of Electromyography, Chinese Society of Neuromuscular Disease. Chinese expert consensus on diagnosis and treatment of subacute combined degeneration. Chinese J Neurol. 2020; 53(4): 269-273.

54. Bikdeli B, Madhavan MV, Jimenez D, Chuich T, Dreyfus I, Driggin E, et al. COVID-19 and thrombotic or thromboembolic disease: implications for prevention, antithrombotic therapy, and followup. J Am Coll Cardiol. 2020; 75(23). doi: 10.1016/j. jacc.2020.04.031

55. Wang D, Hu B, Hu C, Zhu F, Liu X, Zhang J, et al. Clinical characteristics of 138 hospitalized patients with 2019 novel coronavirus-infected pneumonia in Wuhan, China. JAMA. 2020; 323(11): 1061-1069. doi: 10.1001/jama.2020.1585

56. Huang C, Wang Y, Li X, Ren L, Zhao J, Hu Y, et al. Clinical features of patients infected with 2019 novel coronavirus in Wuhan, China. Lancet. 2020; 395(10223): 497-506. doi: 10.1016/S01406736(20)30183-5

57.Zhou F, Yu T, Du R, Fan G, Liu Y, Liu Z, et al. Clinical course and risk factors for mortality of adult inpatients with COVID-19 in Wuhan, China: a retrospective cohort study. Lancet. 2020; 395(10229): 1054-1062. doi: https://doi. org/10.1016/S0140-6736(20)30566-3

58. Violi F, Pastori D, Cangemi R, Pignatelli P, Loffredo L. Hypercoagulation and antithrombotic treatment in coronavirus 2019: A new challenge. Thromb Haemost. 2020; 120(6): 949-956. doi: 10.1055/s0040-1710317

59. Kahn SR, Lim W, Dunn AS, Cushman M, Dentali F, Akl EA, et al. Prevention of VTE in nonsurgical patients. Chest. 2012; 141(2 Suppl): e195S-e226S. doi: $10.1378 /$ chest.11-2296

60. Schünemann HJ, Cushman M, Burnett AE, Kahn SR, Beyer-Westendorf J, Spencer FA, et al. American Society of Hematology 2018 guidelines for management of venous thromboembolism: prophylaxis for hospitalized and nonhospitalized medical patients. Blood Adv. 2018; 2(22): 3198225. doi: 10.1182/bloodadvances.2018022954

61. Moores LK, Tritschler T, Brosnahan S, Carrier M, Collen JF, Doerschug K, et al. Prevention, diagnosis, and treatment of VTE in Patients with coronavirus disease 2019. Chest. 2020; S0012369220316251. doi: 10.1016/j.chest.2020.05.559 